\title{
Adaptive Parallel/Serial Sampling Mechanisms for Particle Filtering in Dynamic Bayesian Networks
}

\author{
Eva Besada-Portas ${ }^{1}$, Sergey M. Plis ${ }^{2,3}$, Jesus M. de la Cruz ${ }^{1}$, and Terran Lane ${ }^{2}$ \\ 1 Universidad Complutense de Madrid, 28040 Madrid, Spain \\ 2 University of New Mexico, Albuquerque NM 87131, USA \\ 3 The Mind Research Network, Albuquerque NM 87106, USA
}

\begin{abstract}
Monitoring the variables of real world dynamical systems is a difficult task due to their inherent complexity and uncertainty. Particle Filters (PF) perform that task, yielding probability distribution over the unobserved variables. However, they suffer from the curse of dimensionality problem: the necessary number of particles grows exponentially with the dimensionality of the hidden state space. The problem is aggravated when the initial distribution of the variables is not well known, as happens in global localization problems. In this paper we present two new adaptive sampling mechanisms for PFs for systems whose variable dependencies can be factored into a Dynamic Bayesian Network. The novel PFs, developed over the proposed sampling mechanisms, exploit the strengths of other existing PFs. Their adaptive mechanisms 1) modify or establish probabilistic links among the subspaces of hidden variables that are independently explored to build particles consistent with the current measurements and past history, and 2) tune the performance of the new PFs toward the behaviors of several existing PFs. We demonstrate their performance on some complex dynamical system estimation problems, showing that our methods successfully localize and track hidden states, and outperform some of the existing PFs.
\end{abstract}

\section{Introduction}

Estimating the hidden state of a multivariate dynamical system remains a large challenge. While the Dynamic Bayesian Network (DBN) formalism provides an excellent way to represent such systems, performing inference in these models is difficult, and approximations are usually necessary. Among the most popular approximate inference methods for DBNs are Particle Filters (PFs): point-mass approximations of the hidden state distribution, which are calculated with sequential Monte Carlo simulation techniques, usually combining importance sampling and weighted resampling steps [1].

In spite of their popularity, PFs can be difficult to apply to new problems. One of the core challenges arises from the familiar curse of dimensionality: in even modest dimensionality spaces, there are high chances that many or most particles will fall into near-zero probability regions of the state space, leading to serious particle depletion and quickly driving the PF off track. This problem is evident, for example, in multi-object tracking tasks [2]. The difficulty is exacerbated when the distribution used to initialize the values of the particles is unlikely to generate particles that have high-probability.

The dimensionality/depletion difficulty can be reduced with a careful choice of the initialization, importance sampling and weighted resampling distributions, but doing so 
is not always straightforward. For example, in global localization problems it is difficult to define a tight initialization proposal distribution. Efficient sampling and resampling proposals developed around specific properties of the dynamics of the problem require extensive domain knowledge and/or generate PFs for specific types of problems [3]4].

The general approaches that have recently appeared in [5]6] overcome some of the inherent difficulties of the standard PF for DBNs [7] using two complementary strategies. The PF of [5] follows the ancestral ordering of the variables to serialize sampling and resampling steps. Whereas, the PFs in [6] parallelize the sampling step to sample different subspaces of hidden variables independently. The serialization in [5] leads to elimination of those particles whose already sampled hidden variables are not probabilistically consistent with some of the measurements. This process avoids sampling their remaining hidden variables. However, it can drive the PF off track when a premature specialization of the survived particles makes it impossible to sample values for the remaining hidden variables consistent with the measurements. The parallelization presented by the authors in [6] lets their PFs create particles that are probabilistically consistent with the measurements associated with each subspace of hidden variables. However, it can continuously reset the PFs when the parallel sampled measurement likely values of their hidden variables are inconsistent with the past history.

The two sampling mechanisms presented in this paper combine the benefits of the previous strategies [567] by automatically adapting the serialization/parallelization level of the proposal distributions used to sample the values of the hidden variables. They are developed within the serial importance sampling methodology and so the developed PFs that incorporate those mechanisms have a firm probabilistic foundation.

The characteristics of the new mechanisms, which represent two new points in the spectrum of already existing sampling strategies, are the following. First, they allow an automatic adaptation of the PF behavior towards the PFs in [5677]. Second, they help to overcome problems of poor particle initialization, improving state localization and convergence when the initial particle sample is far from the target distribution. And third, they need fewer particles than some of the existing methods.

Finally, this paper also analyzes in depth the characteristics of the PFs presented in [5]6/7] and states the old and new techniques under a unified formalism.

\section{Particle Filter Fundamentals}

Our core contribution is the development of two new proposal distributions that support the definition of PFs for DBNs that adapt their behaviors towards the serial PF of [5], the parallel PF of [6], and the standard PF of [7]. In this section we introduce a uniform notation and formalism in which to state the previous and new PFs.

\subsection{Definitions and Notation}

In this paper, a capital letter $U$ represents a random variable, a boldface capital letter $\boldsymbol{U}$ - a set of random variables, a lowercase letter $u$-the specific value of the corresponding random variable $U$, and a lowercase bold letter $\boldsymbol{u}$ - an assignment of the values to the variables of its set $\boldsymbol{U}$. We also define $\mathcal{P}(\boldsymbol{U})$ as a partition of $\boldsymbol{U}$. 
A Bayesian Network (BN) is an annotated directed graph that encodes the probability distribution of a set of random variables $\boldsymbol{V}$. DBNs model the distribution of a sequence of sets of variables. A set of variables belonging to the $k^{t h}$ time slice is represented as $\boldsymbol{V}_{k}$ and the total set of variables up to the current time slice $t$ is $\boldsymbol{V}_{0: t}$. To distinguish hidden and observed variables, we use $X, \boldsymbol{X}_{t}$ and $\boldsymbol{X}_{0: t}$ to denote the former, and $Y, \boldsymbol{Y}_{t}$ and $\boldsymbol{Y}_{0: t}$ for the latter. The graph is completely defined by the sets of parents of all its variables: $\operatorname{Pa}_{k}(V)$ represents the subset of the parents of variable $V$ that belong to time slice $k$ and $\operatorname{pa}_{k}(V)$ their assignment to particular values. Similarly, we also define the set of children of a variable and their assignments by $\mathrm{Ch}_{k}(V)$ and $\operatorname{ch}_{k}(V)$.

Probability distributions will be represented as $\mathrm{p}(\cdot), \mathrm{q}(\cdot)$ and $\mathrm{r}(\cdot)$ : the first related with the probabilities of the problem and the others with the proposal distributions used to sample the values of the particles from. The operation $a \sim \mathrm{q}(\cdot)$ represents sampling $a$ according to $\mathrm{q}(\cdot)$. The operation $E_{\mathrm{p}(X \mid \cdot)}[X]$ represents the expected value of $X$ with respect to $\mathrm{p}(X \mid \cdot)$. And $\delta(\cdot)$ and $\delta_{i}^{j}$ are respectively the Dirac and Kronecher delta functions.

A PF approximates the probability $\mathrm{p}(\boldsymbol{X} \mid \boldsymbol{y})$ of a set of hidden variables $\boldsymbol{X}$ given the values of the measurements $\boldsymbol{Y}$ by the point mass distribution $\sum_{i=1}^{N} w\left(\boldsymbol{x}^{(i)}\right) \delta\left(\boldsymbol{X}-\boldsymbol{x}^{(i)}\right)$, where $\boldsymbol{x}^{(i)}$ are the values of the variables in $\boldsymbol{X}$ in the $i$-th particle, $w\left(\boldsymbol{x}^{(i)}\right)$ their weights, and $N$ the number of particles. Additionally, $x^{(i)}, x_{0: t}^{(i)}$ and $\mathrm{pa}_{k}^{(i)}(V)$ represent the assignments of $X, \boldsymbol{X}_{0: t}$ and $\operatorname{Pa}_{k}(V)$ to the values they have in the $i$-th particle.

Finally, in this paper we only consider DBNs whose variables have parents belonging to the current or previous time slice $\left(\forall V \in \boldsymbol{V}_{t} \wedge \forall k \notin\{t, t-1\} \operatorname{Pa}_{k}(V)=\emptyset\right)$. This restriction upon the structure of the DBN factors the joint probability of the set of hidden and observation variables up to time slice $t$ as Eq. (1) shows.

$$
\begin{gathered}
\mathrm{p}\left(\boldsymbol{X}_{0: t}, \boldsymbol{Y}_{0: t}\right)=\mathrm{p}\left(\boldsymbol{X}_{t}, \boldsymbol{Y}_{t} \mid \boldsymbol{X}_{0: t-1}, \boldsymbol{Y}_{0: t-1}\right) \mathrm{p}\left(\boldsymbol{X}_{0: t-1}, \boldsymbol{Y}_{0: t-1}\right) \\
\mathrm{p}\left(\boldsymbol{X}_{t}, \boldsymbol{Y}_{t} \mid \boldsymbol{X}_{0: t-1}, \boldsymbol{Y}_{0: t-1}\right)=\prod_{X \in \boldsymbol{X}_{t}} \mathrm{p}\left(X \mid \mathrm{Pa}_{t-1}(X), \operatorname{Pa}_{t}(X)\right) \prod_{\boldsymbol{Y} \in \boldsymbol{Y}_{t}} \mathrm{p}\left(Y \mid \operatorname{Pa}_{t-1}(Y), \operatorname{Pa}_{t}(Y)\right)
\end{gathered}
$$

\subsection{Importance Sampling (IS) and Weighted Resampling (WR)}

The values of the particles $\boldsymbol{x}^{(i)}$ and their weights $w\left(\boldsymbol{x}^{(i)}\right)$ used to approximate the probability distribution $\mathrm{p}(\boldsymbol{X} \mid \boldsymbol{y})$ as $\sum_{i=1}^{N} w\left(\boldsymbol{x}^{(i)}\right) \delta\left(\boldsymbol{X}-\boldsymbol{x}^{(i)}\right)$ are obtained by means of a sequential combination of importance sampling and weighted resampling steps [8]. The way of proceeding for each step is detailed in the following subsections, in order to state the serial, parallel, standard and adaptive PFs under a unified perspective. In short, IS is used to create the particles while WR is carried out to increment the number of particles in the regions of high interest and reduce them in the others.

Importance Sampling is used to (1) create new particles by means of a proposal distribution $\mathrm{q}(\boldsymbol{X} \mid \boldsymbol{y})$ that generates their values $\left(\boldsymbol{x}^{(i)} \sim \mathrm{q}(\boldsymbol{X} \mid \boldsymbol{y})\right)$ and (2) calculate their weights as $w\left(\boldsymbol{x}^{(i)}\right) \propto \mathrm{p}\left(\boldsymbol{x}^{(i)} \mid \boldsymbol{y}\right) / \mathrm{q}\left(\boldsymbol{x}^{(i)} \mid \boldsymbol{y}\right)$.

The IS operation can be carried out sequentially exploiting the independence assumptions imposed by the structure of the DBN and therefore the recursive factorization of the joint presented in Eq. (1). The following two IS steps, whose development is detailed in [689], emerge from different choices of posterior distributions $\mathrm{p}(\boldsymbol{X} \mid \boldsymbol{y})$. 
- When $\mathrm{p}\left(\boldsymbol{X}_{0: t} \mid \boldsymbol{y}_{1: t}\right)$ is the posterior distribution to be approximated by the point mass representation $\sum_{i=1}^{N} w\left(\boldsymbol{x}_{0: t}^{(i)}\right) \boldsymbol{\delta}\left(\boldsymbol{X}_{0: t}-\boldsymbol{x}_{0: t}^{(i)}\right)$ the IS step can obtain the values $\boldsymbol{x}_{t}^{(i)}$ (of the hidden variables $\boldsymbol{X}_{t}$ of the current time slice $t$ ) associated with each particle $\boldsymbol{x}_{0: t}^{(i)}$ by means of the proposal $\mathrm{q}\left(\boldsymbol{x}_{t}^{(i)} \mid \boldsymbol{x}_{0: t-1}, \boldsymbol{y}_{1: t}\right)$ and calculate their weights $w\left(\boldsymbol{x}_{0: t}^{(i)}\right)$ with Eq. (2). As this IS step is responsible for estimating the probability of the trajectory $\boldsymbol{X}_{0: t}$ of the hidden variables, we will call it hereafter "Trajectory" IS or TIS.

- When $\mathrm{p}\left(\boldsymbol{X}_{t} \mid \boldsymbol{y}_{1: t}\right)$ is the posterior distribution to be approximated by the point mass representation $\sum_{i=1}^{N} w\left(\boldsymbol{x}_{t}^{(i)}\right) \boldsymbol{\delta}\left(\boldsymbol{X}_{t}-\boldsymbol{x}_{t}^{(i)}\right)$ the IS step can obtain the values $\boldsymbol{x}_{t}^{(i)}$ (of the hidden variables $\boldsymbol{X}_{t}$ of the current time slice $t$ ) of the particle $\boldsymbol{x}_{t}^{(i)}$ by means of the proposal q $\left(\boldsymbol{x}_{t}^{(i)} \mid \boldsymbol{y}_{1: t}\right)$ and calculate their weights $w\left(\boldsymbol{x}_{t}^{(i)}\right)$ with Eq. (3). As this IS step is responsible for estimating the probability of the hidden state $\boldsymbol{X}_{t}$ at the current time slice $t$, we will call it hereafter "Instantaneous" IS or IIS.

$$
\begin{aligned}
w\left(\boldsymbol{x}_{0: t}^{(i)}\right) \propto \frac{\prod_{Y \in \boldsymbol{Y}_{t}} \mathrm{p}\left(y \mid \mathrm{pa}_{t-1}^{(i)}(Y), \mathrm{pa}_{t}^{(i)}(Y)\right) \prod_{X \in \boldsymbol{X}_{t}} \mathrm{p}\left(x^{(i)} \mid \mathrm{pa}_{t-1}^{(i)}(X), \mathrm{pa}_{t}^{(i)}(X)\right)}{\mathrm{q}\left(\boldsymbol{x}_{t}^{(i)} \mid \boldsymbol{x}_{0: t-1}, \boldsymbol{y}_{1: t}\right)} w\left(\boldsymbol{x}_{0: t-1}^{(i)}\right) \\
w\left(\boldsymbol{x}_{t}^{(i)}\right) \propto \frac{\sum_{j=1}^{N}\left(w\left(\boldsymbol{x}_{t-1}^{(j)}\right) \prod_{Y \in \boldsymbol{Y}_{t}} \mathrm{p}\left(y \mid \mathrm{pa}_{t-1}^{(j)}(Y), \mathrm{pa}_{t}^{(i)}(Y)\right) \prod_{X \in \boldsymbol{X}_{t}} \mathrm{p}\left(x^{(i)} \mid \mathrm{pa}_{t-1}^{(j)}(X), \mathrm{pa}_{t}^{(i)}(X)\right)\right)}{\mathrm{q}\left(\boldsymbol{x}_{t}^{(i)} \mid \boldsymbol{y}_{1: t}\right)}
\end{aligned}
$$

Note that both IS use a proposal $\mathrm{q}\left(\boldsymbol{x}_{t}^{(i)} \mid \cdot\right)$ in each time iteration $t$ to sequentially generate only the values $\boldsymbol{x}_{t}^{(i)}$ associated with the hidden variables $\boldsymbol{X}_{t}$ that belong to the time slice $t$. They also obtain the weights of the whole particle $\left(w\left(\boldsymbol{x}_{0: t}^{(i)}\right)\right.$ in TIS and $w\left(\boldsymbol{x}_{t}^{(i)}\right)$ in IIS) based on the values of the weights of the particle up to the previous time slice $\left(w\left(\boldsymbol{x}_{0: t-1}^{(i)}\right)\right.$ in TIS and $w\left(\boldsymbol{x}_{t-1}^{(j)}\right)$ in IIS), and the values of the hidden and observed variables at $t$ and $t-1\left(x_{t}^{(i)}, y, \operatorname{pa}_{t}^{(i)}(V)\right.$ and $\left.\mathrm{pa}_{t-1}^{(k)}(V)\right)$. Finally, in both numerators also appears the joint distribution (introduced in Eq. (1D) of the current time variables given their parents.

The main computational difference, which affects the number of operations of the selected IS step, appears in the numerator of the expressions used to calculate the weights: the IIS Eq. (3) has a summation that doesn't appear in the TIS Eq. (2). The total computational cost also depends on the proposal distribution $\mathrm{q}\left(\boldsymbol{x}_{t}^{(i)} \mid \cdot\right)$ used to sample the values of $\boldsymbol{x}_{t}^{(i)}$, and so, IIS is not necessary computationally more costly than TIS. Moreover, selecting the appropriated proposals both IS steps can be equivalent. Besides, Eq. (3) can be simplified taking out of the summation those variables that don't have hidden parents belonging to the previous time slice $\left(V \in \boldsymbol{V}_{t}\right.$ s.t. $\left.\operatorname{Pa}_{t-1}(V) \cap \boldsymbol{X}_{t-1}=\emptyset\right)$.

It is also important to mention that although the two types of IS estimate the probability of a different set of hidden variables ( $\boldsymbol{X}_{0: t}$ in TIS and $\boldsymbol{X}_{t}$ in IIS), both can be used to estimate the values of the current state variables $\boldsymbol{X}_{t}$, because the trajectory $\boldsymbol{X}_{0: t}$ also 
includes them. However, the search space of TIS is significantly larger, and so the number of necessary particles in IIS should be smaller. Another interesting fact to consider is that TIS can be used to estimate any $\boldsymbol{X}_{k}$ with $k \leq t$ from the approximated distribution $\mathrm{p}\left(\boldsymbol{X}_{0: t} \mid \boldsymbol{y}_{1: t}\right)$ and so the estimated values of $\boldsymbol{X}_{k}$ can take into account the measurements up to $t$. In IIS the values of $\boldsymbol{X}_{k}$ have to be estimated from $\mathrm{p}\left(\boldsymbol{X}_{k} \mid \boldsymbol{y}_{1: k}\right)$ and so they can only take into account the measurements up to $k$.

Weighted Resampling is used to approximate a $\mathrm{p}(\boldsymbol{X} \mid \boldsymbol{y})$ that is already approximated by an existing point-mass representation $\sum_{i=1}^{N} w\left(\boldsymbol{x}^{(i)}\right) \delta\left(\boldsymbol{X}-\boldsymbol{x}^{(i)}\right)$ by another pointmass representation $\sum_{i=1}^{N} w\left(\boldsymbol{x}^{\prime(i)}\right) \delta\left(\boldsymbol{X}-\boldsymbol{x}^{\prime(i)}\right)$. The WR operation works by redistributing the set of existing weighted particles $\left(\boldsymbol{x}^{(i)}, w\left(\boldsymbol{x}^{(i)}\right)\right)$ using a resampling strategy (such as multinomial, systematic or residual sampling) that for each $\boldsymbol{x}^{\prime(i)}$ picks a $\boldsymbol{x}^{(j)}$ according to the $\mathrm{r}\left(\boldsymbol{x}^{(j)}\right)$ values obtained with a selected $\mathrm{r}(\boldsymbol{X})$ function. The weights $w\left(\boldsymbol{x}^{\prime(i)}\right)$ of the new set of particles $\boldsymbol{x}^{\prime(i)}$ are calculated as $w\left(\boldsymbol{x}^{(j)}\right) / \mathrm{r}\left(\boldsymbol{x}^{(j)}\right)$.

The usual election of $\mathrm{r}(\boldsymbol{X})$ in TIS-based PFs is $\mathrm{r}\left(\boldsymbol{x}_{0: t}^{(j)}\right)=w\left(\boldsymbol{x}_{0: t}^{(j)}\right)$ because this WR step applied after TIS eliminates the particles with negligible $w\left(\boldsymbol{x}_{0: t}^{(j)}\right)$ and populates the hidden space $\boldsymbol{X}_{0: t}$ with equally weighted particles distributed according to $w\left(\boldsymbol{x}_{0: t}^{(j)}\right)$. Thus, it focuses the PF to explore those regions that look more promising so far. The same approach can be used in IIS-based PFs making $\mathrm{r}\left(\boldsymbol{x}_{t}^{(j)}\right)=w\left(\boldsymbol{x}_{t}^{(j)}\right)$.

Finally, it is worth mentioning that a combined sequential implementation of IS with the usual WR steps can delete those particles that were so far negligible for the WR step according to $w\left(\boldsymbol{x}_{0: t}^{(j)}\right)$ in TIS and $w\left(\boldsymbol{x}_{t}^{(j)}\right)$ in IIS but whose information could be important taking into account the future measurements $\left(\boldsymbol{y}_{f}\right.$ with $\left.f>t\right)$. For that reason some PFs avoid the WR step or perform it every now and then [10].

\section{General PFs for DBN}

In this section we present the three general PFs whose behavior we want to dynamically approximate with the new adaptive parallel/serial sampling proposals. We also analyze their behavior to justify later the benefits we expect from the new adaptive mechanisms.

\subsection{Standard PF (KLPF)}

The PF presented in [7] (that we will call KLPF after their authors Koller and Lenner) approximates the posterior $\mathrm{p}\left(\boldsymbol{X}_{0: t} \mid \boldsymbol{y}_{1: t}\right)$ using for each time step a TIS step followed by a WR step. The TIS proposal $\mathrm{q}\left(\boldsymbol{x}_{t}^{(i)} \mid \cdot\right)$ is the product of the transition priors presented in Eq. (4) and so the values of each $X$ in $X_{t}$ can be sampled from $\mathrm{p}\left(X \mid \mathrm{pa}_{t-1}^{(i)}(X), \mathrm{pa}_{t}^{(i)}(X)\right)$ using the ancestral ordering of the hidden variables within the time slice $t$. The WR $\mathrm{r}\left(\boldsymbol{X}_{0: t}\right)$ function is the usual choice: $\mathrm{r}\left(\boldsymbol{x}_{0: t}^{(j)}\right)=w\left(\boldsymbol{x}_{0: t}^{(j)}\right)$. So, according to Eq. (2), the selected proposal and WR step, $w\left(\boldsymbol{x}_{0: t}^{(i)}\right)=\prod_{Y \in \boldsymbol{Y}_{t}} \mathrm{p}\left(y \mid \mathrm{pa}_{t-1}^{(i)}(Y), \mathrm{pa}_{t}^{(i)}(Y)\right)$.

$$
\mathrm{q}\left(\boldsymbol{x}_{t}^{(i)} \mid \cdot\right)=\prod_{X \in \boldsymbol{X}_{t}} \mathrm{p}\left(X \mid \mathrm{pa}_{t-1}^{(i)}(X), \mathrm{pa}_{t}^{(i)}(X)\right)
$$


The choices of $\mathrm{q}(\cdot)$ and $\mathrm{r}(\cdot)$ make the TIS generation of the values $\boldsymbol{x}_{t}^{(i)}$ probabilistically consistent with the values of $\boldsymbol{x}_{t-1}^{(i)}$ and the WR selection of the survival particles probabilistically consistent with the current measurements. Once KLPF falls off track the chances to recover are small because the values of the future $x_{f}^{(i)}(f>t)$ will depend on the current off-track $\boldsymbol{x}_{t}^{(i)}$. That is, KLPF is good for building particles $\boldsymbol{x}_{0: t}^{(i)}$ probabilistically consistent firstly with the past hidden history $\boldsymbol{x}_{0: t-1}^{(i)}$ and secondary with the new measurements $\boldsymbol{y}_{t}$.

\subsection{Serial PF (SPF)}

The PF presented in [5] approximates $\mathrm{p}\left(\boldsymbol{X}_{0: t} \mid \boldsymbol{y}_{1: t}\right)$ dividing the operations of each time step $t$ in a serial of alternating partial TIS and WR steps. The alternation is imposed by the ancestral ordering of the variables $V \in \boldsymbol{V}_{t}$ : when $V$ is hidden $v^{(i)} \sim$ $\mathrm{p}\left(V \mid \mathrm{pa}_{t-1}^{(i)}(V), \mathrm{pa}_{t}^{(i)}(V)\right)$, when $V$ is observed the particles built up to the moment are weighted resampled according to $\mathrm{p}\left(v \mid \mathrm{pa}_{t-1}^{(i)}(V), \mathrm{pa}_{t}^{(i)}(V)\right)$.

Overall SPF uses the same $\mathrm{q}\left(\boldsymbol{x}_{t}^{(i)} \mid \cdot\right)$ as KLPF with WR steps based on the likelihood of each $Y \in \boldsymbol{Y}_{t}$ between the sampling of the $X \in \boldsymbol{X}_{t}$ according to the transition priors. Each partial TIS makes the sampled $\boldsymbol{x}^{(i)}$ probabilistically consistent with the already sampled hidden variables. Each partial WR selects the particles which are probabilistically consistent with the given $y$. Thus, SPF is good for building particles $\boldsymbol{x}_{0: t}^{(i)}$ alternatively probabilistically consistent with the already sampled hidden history and used measurements. SPF will also fall offtrack easily when the likelihood of each measurement and the product of the likelihoods take significantly different values.

\subsection{Parallel PFs (PPF)}

The PFs described in [6] approximate either $\mathrm{p}\left(\boldsymbol{X}_{0: t} \mid \boldsymbol{y}_{1: t}\right)$ or $\mathrm{p}\left(\boldsymbol{X}_{t} \mid \boldsymbol{y}_{1: t}\right)$ using for each time step respectively a TIS or IIS step, followed optionally by a WR step. The TIS or IIS proposal $\mathrm{q}\left(\boldsymbol{x}_{t}^{(i)} \mid \cdot,\right)$ is presented in Eq. (5) and (6). It groups the $X \in \boldsymbol{X}_{t}$ in subsets $\boldsymbol{X}$ belonging to a defined 1 disjoint partition $P\left(X_{t}\right)$. And it independently samples the values of the hidden variables within each subset $\boldsymbol{X}$ from a mixture model of the product of transition priors where each mixture component is weighted with the product of the expected likelihoods 2 of the measurements $Y$ associated with the $X \in X$. When applied, the $\operatorname{WR~} \mathrm{r}(\cdot)$ function is the usual choice: $\operatorname{r}\left(\boldsymbol{x}_{0: t}^{(j)}\right)=w\left(\boldsymbol{x}_{0: t}^{(j)}\right)$ or $\mathrm{r}\left(\boldsymbol{x}_{t}^{(j)}\right)=w\left(\boldsymbol{x}_{t}^{(j)}\right)$.

$$
\mathrm{q}\left(\boldsymbol{x}_{t}^{(i)} \mid \cdot\right)=\prod_{\boldsymbol{X} \in \mathcal{P}\left(\boldsymbol{X}_{t}\right)}\left(\sum_{j=1}^{N}\left(\alpha_{j}^{\boldsymbol{X}} \prod_{X \in \boldsymbol{X}} \mathrm{p}\left(x \mid \mathrm{pa}_{t-1}^{(j)}(X), \mathrm{pa}_{t}^{(i)}(X)\right)\right)\right)
$$

${ }^{1}$ The partition is constructed based on the DBN structure with the rules presented in [6].

${ }^{2}$ While the value of any hidden $X \in \mathrm{Pa}_{t}(V)$ in $\mathrm{pa}_{t}^{(j)}(V)$ is the value of $X$ in the $j$-th particle, the value associated with the same $X \in \mathrm{Pa}_{t}(V)$ in $\mathrm{pa}_{t}^{*(j)}(V)$ is $E_{p\left(X \mid \mathrm{pa}_{t-1}^{(j)}(X), \mathrm{pa}_{t}^{*(j)}(X)\right)}[X]$. The expectations are needed because the value of $X$ won't be sampled until the component of the mixture is selected according to $\alpha_{j}^{X}$. 


$$
\alpha_{j}^{\boldsymbol{X}} \underset{Y \in \mathrm{Ch}_{t}(X) \wedge X \in X}{ } \mathrm{p}\left(y \mid \mathrm{pa}_{t-1}^{(j)}(Y), \mathrm{pa}_{t}^{*(j)}(Y)\right)
$$

The choice of $\mathrm{q}(\cdot)$ makes the IS generation of the values $\boldsymbol{x}_{t}^{(i)}$ probabilistically consistent with the expected likelihood of the measurements associated with each of the disjoint subsets of hidden variables $\boldsymbol{X}$ defined by $\mathcal{P}\left(\boldsymbol{X}_{t}\right)$. However, the independence imposed by the factorization of the proposal can lead to creation of particles that are not consistent with the previous time step particles $\left(\boldsymbol{x}_{0: t-1}^{(i)}\right.$ or $\left.\boldsymbol{x}_{t-1}^{(i)}\right)$. When no particle is consistent, the numerators of Eq. (2) or Eq. (3) will make all the weights zero and the PPF will be automatically reset. This behavior is positive when the PPF is losing track but unnecessary when that is not the case. So the PPFs are good for building particles $\left(\boldsymbol{x}_{0: t}^{(i)}\right.$ or $\boldsymbol{x}_{t}^{(i)}$ ) probabilistically consistent firstly with different subsets of current measurements and secondly with the previous time step particles $\left(\boldsymbol{x}_{0: t-1}^{(i)}\right.$ or $\left.\boldsymbol{x}_{t-1}^{(i)}\right)$.

The choice of $\mathrm{r}(\cdot)$ influences the elimination of particles with negligible weights by WR. However, this choice also decrements the sampling possibilities of the independent mixture proposals. So disabling the WR step increases the PPFs sampling possibilities facilitating the PPFs reset when the measurements subsets contradict the hidden history.

\section{Adaptive Sampling Mechanisms for DBN}

Optimal IS steps are those that minimize the variance of the weights [10]. In the design of PFs for DBNs this requirement implies that the sampling proposal $\mathrm{q}\left(\boldsymbol{x}_{t}^{(i)} \mid \cdot\right)$ samples the new values of $\boldsymbol{X}_{t}$ simultaneously taking into account the values of $\boldsymbol{x}_{t-1}^{(i)}$ and $\boldsymbol{y}_{t}$. However, this is rarely possible and so non-optimal proposals are usually selected.

The sampling proposals used in KLPF, SPF and PPFs are not optimal. Nevertheless, these proposals exhibit several complementary positive probabilistic behaviors. KLPF and SPF build particles probabilistically consistent firstly with $\boldsymbol{x}_{t-1}^{(i)}$ and secondly with the current measurements $\boldsymbol{y}_{t}$, while the PPFs build particles probabilistically consistent firstly with $\boldsymbol{y}_{t}$ and secondly with $\boldsymbol{x}_{t-1}^{(i)}$. Besides, KLPF builds particles consistent with all the current measurements $\boldsymbol{y}_{t}$, SPF builds particles incrementally consistent with each measurement $y \in \boldsymbol{y}_{t}$, and PPFs build them probabilistically consistent with subsets of measurements and of hidden variables.

We believe that a PF that simultaneously takes advantage of several of those behaviors should be closer to the optimal PF. Based on that intuition we have developed two novel sampling mechanisms that adapt the proposal towards the behavior of some of the general PFs while the PF is running. In the following section we present the adapting proposals, the mechanisms that control the adaptation and the PFs based on these two.

\subsection{Adaptive Proposals}

In this section we present two proposals that let the PFs based on them adapt the behavior towards two different types of PFs. Both are based on the adaptation of the parallel proposa 3 of Eq. (5) towards the behavior obtained in KLFP or SPF by the simultaneous use of TIS based on the proposal of Eq. (4) and WR based on the values of the weights.

${ }^{3}$ We adapt the parallel proposal toward the other behaviors due to its higher complexity. 
Grouped Parallel Proposal: The parallel proposal of Eq. (5) divides the $\boldsymbol{Y}_{t}$ used to pick the values $\boldsymbol{x}_{t-1}^{(i)}$ to sample from the disjoint subsets $\boldsymbol{X}$ according to a given $\mathcal{P}\left(\boldsymbol{X}_{t}\right)$. The $\mathcal{P}\left(\boldsymbol{X}_{t}\right)$ definition is not unique, but it must at least fulfill a set of restrictions imposed by the DBN structure [6]. The basic disjoint subsets of $\boldsymbol{X} \in \boldsymbol{X}_{t}$ and $\boldsymbol{Y} \in \boldsymbol{Y}_{t}$ imposed by the DBN structure and defined by the partitions, named hereafter as $\mathcal{P}^{D B N}\left(\boldsymbol{X}_{t}\right)$ and $\mathcal{P}^{D B N}\left(\boldsymbol{Y}_{t}\right)$, can be grouped to define new pairs of partitions $\left(\mathcal{P}^{\prime}\left(\boldsymbol{X}_{t}\right), \mathcal{P}^{\prime}\left(\boldsymbol{Y}_{t}\right)\right)$.

As different pairs of partitions modify the behavior of the parallel proposal presented in Eq. (5), the grouping of the existing subsets to define new partitions and the use of the different partition pairs $\left(\mathcal{P}\left(\boldsymbol{X}_{t}\right), \mathcal{P}\left(\boldsymbol{Y}_{t}\right)\right)$ in each time step of the PPFs makes the PPFs achieve a dynamic sampling behavior.

This simple idea is exploited by our first adaptive proposal. Originally we use the proposal of Eq. (5) with the pair $\left(\mathcal{P}^{D B N}\left(\boldsymbol{X}_{t}\right), \mathcal{P}^{D B N}\left(\boldsymbol{Y}_{t}\right)\right)$, and in a posterior time step we join two of the subsets of the previous partition pair to define a new pair $\left(\mathcal{P}^{\prime}\left(\boldsymbol{X}_{t}\right), \mathcal{P}^{\prime}\left(\boldsymbol{Y}_{t}\right)\right)$ to be used in Eq. (5). The grouping adaptation can continue until all the sets are joined and the final possible pair $\left(\mathcal{P}^{F}\left(\boldsymbol{X}_{t}\right)=\boldsymbol{X}_{t}, \mathcal{P}^{F}\left(\boldsymbol{Y}_{t}\right)=\boldsymbol{Y}_{t}\right)$ is reached. Or stopped to restart the process from the pair $\left(\mathcal{P}^{D B N}\left(\boldsymbol{X}_{t}\right), \mathcal{P}^{D B N}\left(\boldsymbol{Y}_{t}\right)\right)$ when the mechanism that controls the adaptation considers it beneficial.

This adaptive proposal makes a PPF based on $\left(\mathcal{P}^{D B N}\left(\boldsymbol{X}_{t}\right), \mathcal{P}^{D B N}\left(\boldsymbol{Y}_{t}\right)\right)$ adapt its behavior toward the KLPF. This is due to the fact that by each grouping step we make a subset of hidden and observation variables bigger and sample the hidden variables within that subspace according to its associated measurements. In the limit it samples all the current hidden values from the same previous particle and picks the selected ones according to the product of likelihoods 4 of all the measurements.

Linked Serial Proposal: Another way of adapting the behavior of the parallel proposal of Eq. (5) consists of imposing an ordering $\left\{\boldsymbol{O}_{1}, \boldsymbol{O}_{2}, \ldots, \boldsymbol{O}_{\left|\mathcal{P}\left(\boldsymbol{X}_{t}\right)\right|}\right\}$ on the subsets of hidden variables defined by the partition $\left(\boldsymbol{O}_{k}=\boldsymbol{X} \in \mathcal{P}\left(\boldsymbol{X}_{t}\right)\right)$, and sample the hidden variables within each subset following that order and a mixture model of the product of the prior transitions whose weights take into account the product of the expected likelihoods and which particles were sampled in the previous subsets. This way of proceeding, that links the chances of picking the elements of a particle with the already selected ones, is defined by Eq. (7) and (8), where $j_{z}$ represent the particle selected for the same particle in the previous subsets, $\alpha_{j}^{\boldsymbol{O}_{k}}$ is the product of expected likelihoods defined in (6), and $\gamma \in[0,1]$ the proposal parameter used to modify the linking level.

$$
\begin{gathered}
\mathrm{q}\left(\boldsymbol{x}_{t}^{(i)} \mid \cdot\right)=\prod_{k=1:\left|\mathcal{P}\left(\boldsymbol{X}_{t}\right)\right|}\left(\sum_{j=1}^{N}\left(\beta_{j}^{\boldsymbol{O}_{k}} \prod_{X \in \boldsymbol{O}_{k}} \mathrm{p}\left(x \mid \mathrm{pa}_{t-1}^{(j)}(X), \mathrm{pa}_{t}^{(i)}(X)\right)\right)\right) \\
\beta_{j}^{\boldsymbol{O}_{k}}=\gamma \alpha_{j}^{\boldsymbol{O}_{k}}+\frac{(1-\gamma)}{k} \sum_{z=1: k-1} \delta_{j}^{j_{z}}
\end{gathered}
$$

This adaptive proposal makes a PF based on it adapt its behavior from PPF to SPF and vice versa. This is due to the fact that when $\gamma=1$ the proposal behaves as the original parallel proposal, and as the values of $\gamma$ is decreased the particles used to sample

\footnotetext{
${ }^{4}$ There is a small additional difference: this new proposal based on Eq. (5) uses the "expected" likelihoods $\mathrm{p}\left(y \mid \mathrm{pa}_{t-1}^{(j)}(Y), \mathrm{pa}_{t}^{*(j)}(Y)\right)$ while KLPF uses the actual ones $\mathrm{p}\left(y \mid \mathrm{pa}_{t-1}^{(j)}(Y), \mathrm{pa}_{t}^{(j)}(Y)\right)$.
} 
the hidden variables within each $\boldsymbol{O}_{k}$ has a higher dependency on which particles were already used to sample the previously sampled subset of hidden variables. As the subsets of observed variables used to calculate the $\alpha_{j}^{X}$ don't change, this adaptive proposal samples the values within each subset taking into account the different subsets of observations and so it makes the PFs based on it behave more closely to SPF than to KLPF. In the limit, when $\gamma=0$, the only set of measurements considered to select the particles is that associated with the first subspace of the ordering, ignoring the effect of the rest.

\subsection{Adaptive Control Mechanism}

The PFs based on the two adapting proposals presented in the previous section need a mechanism that modifies their behavior by means of the grouping process used in the first case or the linking parameter $\gamma$ in the second. The current mechanisms consist of:

1. Initializing the new proposals to work according to the original parallel one. That is, the grouped parallel proposal uses Eq. (5) with $\left(\mathcal{P}^{D B N}\left(\boldsymbol{X}_{t}\right), \mathcal{P}^{D B N}\left(\boldsymbol{Y}_{t}\right)\right)$ and the linked serial proposal uses Eq. (7) with $\left(\mathcal{P}^{D B N}\left(\boldsymbol{X}_{t}\right), \mathcal{P}^{D B N}\left(\boldsymbol{Y}_{t}\right)\right)$ and $\gamma=1$.

2. In each time step of the PF, if there is a $w(\cdot) \neq 0$, the proposal adapts its behavior away from the original parallel one. In the grouped parallel proposal two of the current independent subsets are picked randomly and joined. In the linked serial proposal the ordering of the subsets is randomly determined and $\gamma$ is decremented a selected amount $\Delta \gamma$ up to a selected threshold $\gamma_{\min }$.

3. In each time step of the PF, if all $w(\cdot)=0$, the proposals are reset to its original value (step 1).

The insight behind the selected mechanism is the following. On one hand, starting with the original parallel proposal and returning to it when the PF have just lost track $(w(\cdot)=$ 0 ) increments the chances to start from a better initial distribution or recover based on the measurements. On the other hand, moving away from the original parallel proposal when the PF is keeping track lets it increase their capacity of being probabilistically consistent with the past history. The random selection of the sets to group or the ordering of the existing sets is used to avoid prioritizing some possibilities over others.

\subsection{The Complete Adaptive Particle Filters}

The first step, before carrying out the filtering steps of the novel PFs, consists of 1) defining the disjoint partitions $\left.\left(\mathcal{P}^{D B N}\left(\boldsymbol{X}_{t}\right), \mathcal{P}^{D B N}\left(\boldsymbol{Y}_{t}\right)\right), 2\right)$ creating the original particles $x_{0}^{(i)}$ using an initialization proposal, and 3) setting up the original values of the selected proposal (either Eq. (5) and (6) with $\mathcal{P}^{D B N}\left(\boldsymbol{X}_{t}\right)$, or Eq. (7) and (8) with a random ordering $\boldsymbol{O}_{k}=\boldsymbol{X} \in \boldsymbol{P}^{D B N}\left(\boldsymbol{X}_{t}\right)$ and $\left.\boldsymbol{\gamma}=1\right)$. Next, and in each time step $t$ :

1. The PF samples the values of the hidden variables $\boldsymbol{x}_{t}^{(i)}$ using one of the new proposals as its currently setup. In short, we calculate either in parallel $\alpha_{j}^{X}$ or sequentially $\beta_{j}^{\boldsymbol{O}_{r}}$ and use those values to select a component of the mixtures and sample the hidden values associated with that component from the product of transition priors.

2. The PF calculates the $w(\cdot)$ of each particle using either Eq. (2) or (3) depending on what posterior we are approximating and Eq. (5) or (7) depending on what proposal 
we are using. The numerator can be zero when the values of the hidden variables in the current time slice are non probabilistically consistent with the ones of the previous time slice and/or the measurements. The denominator can't, because we have generated it with the proposal. So, the final $w(\cdot) \geq 0$.

3. If any $w(\cdot)>0$ then adapt the selected proposal away from the original parallel one. In the grouped parallel proposal two of the current independent subsets are picked randomly and joined. In the linked serial proposal the next ordering of the subsets is randomly determined and $\gamma$ is decremented by a selected amount $\Delta \gamma$ up to a selected threshold $\gamma_{\min }$. Go to step 1 .

4. If all $w(\cdot)=0$, the proposals are reset to its original value $\left(\mathcal{P}^{D B N}\left(\boldsymbol{X}_{t}\right)\right.$ in the grouped parallel and $\gamma=1$ in the linked serial). Go to step 1 .

The computational cost of the new adaptive PFs depends on the expressions used for calculating the numerator (TIS or IIS) and denominator (group parallel or linked serial proposal) of (2) or (3). The new TIS PFs need less computation than their IIS counterparts. The corresponding PPFs need more/less computations than the ones with the grouping/linked proposals. KLPF and SPF are less computational demanding.

\section{Related Work}

Our novel PFs, called GPF and LPF after their "Grouping” and 'Linked" sampling proposals, represent new points in the spectrum of Monte Carlo sequential importance sampling strategies that adapt their behavior from the parallel PF in [6] to either the standard PF in [7] or the serial PF in [5], as Fig. 1 represents.

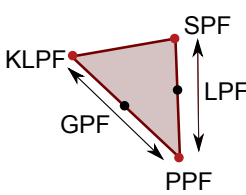

Fig. 1. Relationships among the behaviors of the different PFs

They are not the first adaptive PFs as there exist others that dynamically change the number of particles [11|12|13], change the transition model $\mathrm{p}\left(X \mid \mathrm{pa}_{t-1}^{(i)}(X), \mathrm{pa}_{t}^{(i)}(X)\right)$ [13 14], enable and disable the WR step [10]13], or use a WR step with an $\mathrm{r}(\cdot)$ function whose values are adapted/smoothed with the previous time step weights [15[16]. Our approach is different: we make use of adaptive sampling proposals controlled by an adaptive mechanism to modify the novel PF behaviors towards several general PFs.

Finally, the idea of using a proposal that samples form the past history and current measurements simultaneously has also been used in the mixture Monte Carlo PF [17], which can't be generally used because sampling new values from the current measurements is not always possible. 


\section{Experimental Results}

The following results compare the new PFs (GPF and LPF with $\Delta \gamma=0.05$ and $\gamma_{\text {min }}=$ 0.1 ) with KLPF, SPF and PPFs using two sets of experiments. The first set, performed with simulated data compare the performance of the PFs using the Root Mean Square Error (RMSE) between the mean value of the particle estimates for each PF and the true value of the hidden variables at the last step of the simulation. In the second, carried out with real world data, we use the PFs to score the structure of a DBN given the measurements. In both cases, the PFs with parallel sampling possibilities (PPF, GPF, LPF) outperform the rest (KLPF and SPF) on average. Besides, the novel GPF and LPF and the old PPF can change their relative performance based on the task.

\subsection{Simulated Experiments}

This section compares all the PFs, working with a limited number of particles, in two complex real problems modeled by different DBNs. We have selected them because the structures of their DBNs, with multiple hidden variables that can be sampled in parallel, let them benefit significantly from PFs with parallel sampling possibilities. In both cases, we want to localize a set of $G$ mobile objects given the information provided by the existing sensors. To be able to distinguish the variables related with the $l$-th object, in this section some variable names have a subindex $\left(V_{l}\right)$.

In the first problem, each mobile object $\left(M_{l}\right)$ is repelled from another $\left(M_{k}\right)$ by a common unknown force $(F)$, and the position of each mobile is observed by a different sensor $\left(S_{l}\right)$. The PFs have to estimate the probability of the hidden variables $\left(M_{l}, F\right)$ given the measurements $\left(S_{l}\right)$ for the DBN whose structure 5 is defined by $\operatorname{Pa}_{t-1}\left(M_{l}\right)=$ $\left\{M_{l}, M_{\text {rem }(l, G)+1}, F\right\}, \operatorname{Pa}_{t-1}(F)=\{F\}$ and $\operatorname{Pa}_{t}\left(S_{l}\right)=\left\{M_{l}\right\}$. The structure of this DBN lets us divide the hidden variables in $G+1$ groups, each with a hidden variable, and assign to the $\alpha_{j}^{\boldsymbol{X}}$ of each group the estimated likelihoods of the measurement associated with each hidden variable $\left(\alpha_{j}^{\{F\}} \propto 1\right.$ and $\left.\alpha_{j}^{\left\{M_{l}\right\}} \propto \mathrm{p}\left(s_{l} \mid \mathrm{pa}_{t}^{*(j)}\left(S_{l}\right)\right)\right)$. The difficulty of this problem originates from the high coupling of the $M_{l}$ variables imposed by $\operatorname{Pa}_{t-1}\left(M_{l}\right)$.

The second problem is an abstraction of a sea rescue problem where an Unmanned Air Vehicle (UAV) has to track several objects that are in the water and whose initial positions are not completely known. The objects $\left(M_{l}\right)$ move with the direction of the sea current and wind $(E)$ and the sensors, which are onboard the UAV $(U)$, are only able to detect $\left(D_{l}\right)$ the mobiles that are within a circular area of the UAV and provide their position $\left(S_{l}\right)$ when detected. The PFs have to estimate the probability of the hidden variables $\left(M_{l}, E\right)$ given the observed ones $\left(U, D_{l}, S_{l}\right)$ for the DBN whose structure is defined by $\mathrm{Pa}_{t-1}\left(M_{l}\right)=\left\{M_{l}, E\right\}, \mathrm{Pa}_{t-1}(E)=\{E\}, \mathrm{Pa}_{t}\left(D_{l}\right)=\left\{M_{l}, U\right\}$ and $\mathrm{Pa}_{t}\left(S_{l}\right)=\left\{M_{l}, D_{l}\right\}$. Again, the structure of this DBN let us divide the hidden variables in $G+1$ groups, each with a hidden variable, and assign to the $\alpha_{j}^{X}$ of each group the product of the estimated likelihoods of the measurements associated with each hidden variable $\left(\alpha_{j}^{\{E\}} \propto 1\right.$ and $\left.\alpha_{j}^{\left\{M_{l}\right\}} \propto \mathrm{p}\left(d_{l} \mid \mathrm{pa}_{t}^{*(j)}\left(D_{l}\right)\right) \mathrm{p}\left(s_{l} \mid \mathrm{pa}_{t}^{*(j)}\left(S_{l}\right)\right)\right)$. This problem is less coupled than the previous, but it is also difficult: it has multiple measurements per hidden variable and when the object is outside the circular area its position is not observed.

\footnotetext{
${ }^{5}$ In the following, $\operatorname{rem}(a, b)$ represents the remainder of dividing $a$ by $b$.
} 
For each problem, we obtain the observations and the real values of the hidden variables from a simulation based on its probability model. Then we run each PF with a given number of particles and 50 different sets of initial particles (the same for each PF to compare their behavior under the same initial conditions). And finally, we measure at the last step of the simulation the performance of each PF using the Root Mean Square Error (RMSE) between the mean value of the particle estimates and the true value.

The complete experiment setup consists of running for each problem the 8 PFs with 3 different number of particles, 3 different initialization region proposals and the same 50 sets of initial particles. The 8 PFs configurations are: KLPF, "Instantaneous" GPF, “Trajectory” GPF, "Instantaneous” LPF, “Trajectory” LPF, "Instantaneous" PPF, “Trajectory" PPF, and SPF. The tested number of particles $N$ are 20, 50, 100. The 3 initialization proposals are uniform rectangular regions of different sizes around the actual initial value: the second quadruples the area of the first, and the third quadruples the area of the second. Each of the 50 sets of initial particles is created for each problem for a combination of initialization proposal and number of particles.

Figure 2 shows the results as ratios of the RMSE obtained by each PF (x-axis) and the RMSE obtained by the "Instantaneous" PPF for each problem (Fig. 2a and Fig. 2b), number of particles (figure row), initialization region (figure column) and initial particle set (y-axis). Mid-grey (orange) ratios represent the equality. They are shown as reference in the sixth column of each figure as the ratio of RMSE( "Instantaneous" PPF)/RMSE( "Instantaneous" PPF)=1. Darker gray (red) shows that RMSE of the "Instantaneous" GPF is better and lighter (yellow) that it is worse. Ratios bigger that 10 or smaller than $1 / 10$ are represented in black and white, and the difference between ratios $>1$ and ratios $<1$ is easily observed by not using the gray levels (colors) around the mid-gray (orange) one.

As the ratios in Fig.2 2 show the improvement with respect to the "Instantaneous" PPF, we can't quantify how well each of the PFs is actually doing. This information appears in Fig. 3 that divides the results of each PF in each configuration in "convergent" (white) and "divergent" (black) based on their RMSE values and a fixed threshold.

Fig. 2 shows that the selected PF usually outperforms, many times with ratios $>10$, KLPF (first dark column) and SPF (last dark column) in both problems and all configurations. The same happens when any of the PFs with parallel sampling possibilities is selected: KLPF and SPF rarely outperform GPF, LPF, and PPF. The worst behavior of KLPF and SPF with respect to the others is also illustrated in Fig. 3, where the results of KLPF and SPF are also usually classified as divergent.

The relations among the PFs with parallel sampling possibilities change with the problem and number of particles: For problem 1, Fig. 2a shows that the PFs compete: the LPFs (columns 4 and 5) are usually worst, and the GPF (columns 2 and 3) behavior improves as we increment the number of particles and decrement the area of the initialization proposal. This behavior is not followed by 20 particles and the third initialization proposal configuration. However, many of the solutions are divergent in the corresponding graphic in Fig. 3a and for that reason we don't consider the ratios that meaningful in that case. For problem 2, Fig. 2b shows that the adaptive PFs are usually better than the original PPF. Moreover, the behavior of GPF is maintained through all the configurations, while the behavior of the LPF improves with the number of particles. 


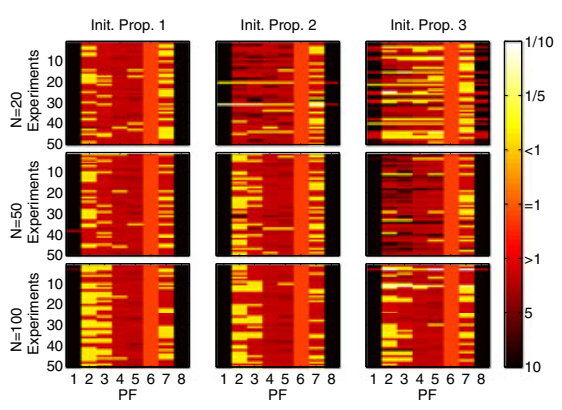

(a) Problem 1

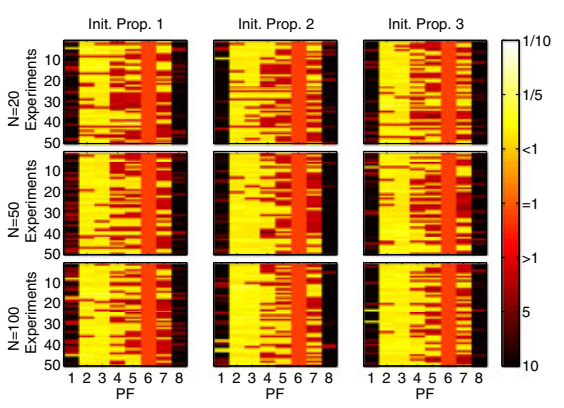

(b) Problem 2

Fig. 2. Ratios of the RMSE of each PF and the RMSE of the "Instantaneous" PPF. In the x-axis, 1 is KLPF, 2 -"Instantaneous" GPF, 3 - "Trajectory" GPF, 4 - "Instantaneous" LPF, 5 -"Trajectory" LPF, 6 - "Instantaneous" PPF, 7 - "Trajectory" PPF, and 8 - SPF

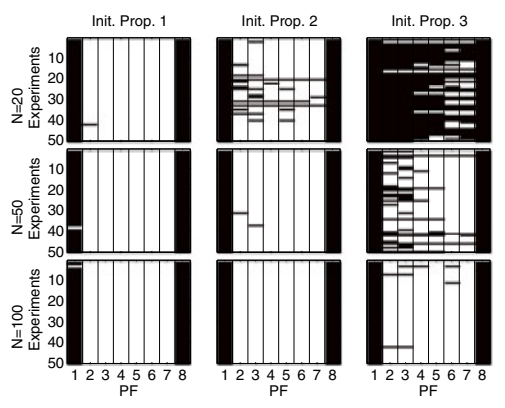

(a) Problem 1

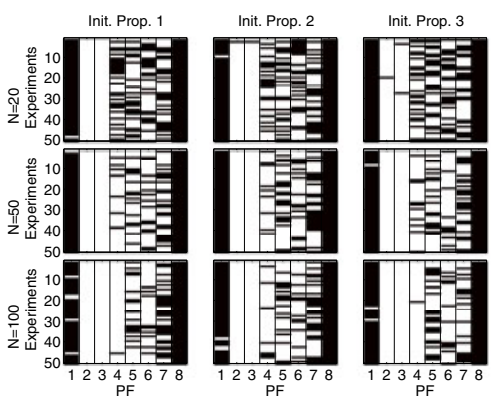

(b) Problem 2

Fig. 3. "Convergent" (white) and "divergent" (black) experiments for all the PFs

Finally, Fig. 3 shows that GPF usually converges (and does it more often than the others except for problem 1 with small number of particles and wider initialization proposal), and that LPF and SPF have similar convergent numbers of experiments.

So, for the selected problems GPF is usually the best (except in problem 1 with just a few particles and extremely wide initialization regions), followed in problem 1 by PPF and in problem 2 by sometimes PPF and others LPF. Besides, the number of convergent runs in a configuration for the adaptive PFs is rarely worse than for the fixed PPF, and so, although the latter can obtain punctually better values, overall the adaptive PFs produce a convergent run at least as often as the PPF.

\subsection{Real Data}

For the real data experiment we have used a functional Magnetic Resonance Imaging (fMRI) dataset [18]. FMRI signal represents the Blood Oxygenation Level Dependent (BOLD) response measured in a small rectangular region of the brain (voxel). BOLD 


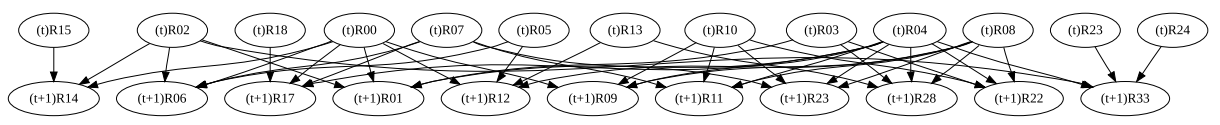

Fig. 4. The network for a subset of regions of interest (ROIs) from the Talairach anatomical atlas database. ROI names are replaced by short labels since they are not significant for our work. Note that each ROI is observed through its indirect measurement by fMRI.

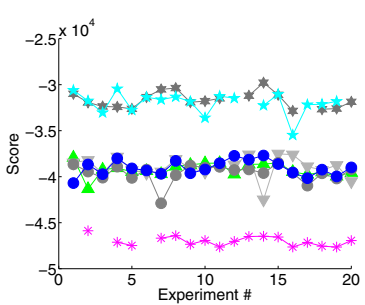

(a) 20 particles

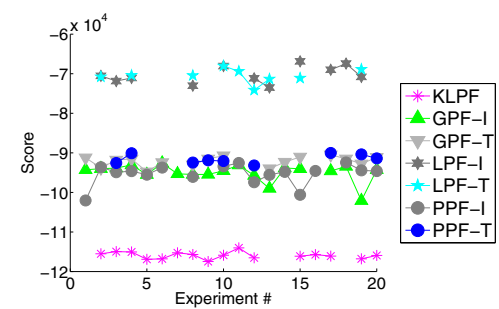

(b) 50 particles

Fig. 5. Cross validation log likelihood score plots for the PFs. Not displayed points mean that the kernel density estimator did not produce a valid result.

response is itself governed by the underlying hidden neural activity. To model generation of the BOLD signal from neural activity we have used the hemodynamic forward model based on a coupled system of ordinary differential equations [19].

The number of voxel measurements collected per time point in a typical fMRI experiment is too large to model directly. Thus voxel time courses were averaged on a per Region of Interest (ROI) basis. ROIs were selected according to the widely used Talairach database [20].

In order to obtain the underlying structure of the DBN, we have used the approach that treats fMRI data as fully observed and quantizes it into categorical representation [18]. Among the discovered DBN families we have used several most significant ones according to the cross validation procedure ( $t$-test with $p$-value of 0.05$)$. This resulted in a DBN of 42 hidden variables per slice. Figure 4 shows a small portion of the hidden structure of the DBN we use. The observation nodes are not shown.

All PFs were run on this dataset for 50 time points (100 seconds with 2 seconds fMRI sampling rate). Since the ground truth for neural activity of ROIs is unknown in this dataset, for evaluation we have used cross validation log likelihood based on kernel density estimators with Gaussian kernels and automatically chosen variance value using a cross validation procedure [21].

The result 6 for 20 runs using 20 and 50 particles are shown in Figure 5. LPFs (stars) show higher score (better), followed by GPFs (triangles) and PPFs (circles). KLPF (asterisks) has considerably lower mean score. Note how the score decreases as the number of particles grows. This is an expected behavior with Parzen window estimators, that is

\footnotetext{
${ }^{6} \mathrm{SPF}$ is missing because the kernel density estimator didn't produce valid results.
} 
due to the smoother and generally wider estimates in the cases of more data that lead to lower probability values.

In part these results support the observations obtained on simulated data: the PFs with parallel sampling possibilities (GPFs, LPFs, and PPFs) have improved performance over the other tested PFs. However, as Figure 5 shows, the adaptive LPFs outperform GPFs and PPFs. This means that the benefit of the use of adaptive PFs depends on the problem. Besides, in this case, the PPF performance can be considered a lower bound of GPFs and LPFs, because the results of the adaptive PFs are not usually worse than the results of the fixed PPFs.

\section{Conclusions}

We present a set of adaptive PFs to estimate the trajectory or current state of the hidden variables of a DBN. They use two adaptive proposals to sample in parallel/serial the values of changing/static-linked subsets of hidden variables to build a whole particle whose weight is updated according to the proposal and the estimation problem.

Our PFs explore different subspaces of the state space while performing the sampling step, and the whole space as a block while updating the weights. In the grouping parallel proposal the variables in the subsets change dynamically while in the linked serial proposal the variables are maintained and the subsets randomly linked. Each proposals is used to develop two different PFs that adapt their behavior from the parallel $\mathrm{PF}$ in [6] to the standard PF in [7] or the serial PF in [5].

The tests used to compare the new and the old PFs show that, with a reduced number of particles when the particles are initialized in spread regions, all the PFs with parallel sampling possibilities (the novel PFs and the old parallel PF) usually outperform the standard and serial PF. Besides, the new PFs usually demonstrate a behavior similar or better than the parallel PF. The different behavior of the adaptive PFs in the different problems implies that different adaptive PFs are good for different DBNs. Determining the best method for each DBN type requires further study which is left for future work.

Finally, it is worth mentioning that the results of the adaptive PFs also depend on their adaptation mechanism. The one that we are testing so far moves more slowly from the parallel PF to the others than the other way around (it resets completely). Besides, we decide the direction of the movement only taking into account if there is at least one particle whose weight is not zero. Although it is enough for letting the novel PFs outperform the already existing PFs, we are planning to explore the use of betterbalanced and softer decision (for instance, based in the number of effective particles [10]) mechanisms in our future work.

\section{Acknowledgments}

This work was supported by the Spanish Grant DPI2009-14552-C02-01. Further, Dr. Besada-Portas was supported by the Spanish post-doctoral Grant EX-2007-0915, Dr. Plis by NIH 1 R01 EB 006841, 1 R01 EB 005846, and Dr. Lane by NSF Grant IIS0705681. The authors also thank the Aula Sun-UCM for providing access to their computational resources for doing parts of the experiments. 


\section{References}

1. Doucet, A., Freitas, N., Gordon, N. (eds.): Sequential Monte Carlo methods in practice. Springer, Heidelberg (2001)

2. MacCormick, J., Isard, M.: Partitioned sampling, articulated objects, and interface-quality hand tracking. In: Vernon, D. (ed.) ECCV 2000. LNCS, vol. 1843, pp. 3-19. Springer, Heidelberg (2000)

3. Doucet, A., de Freitas, N., Murphy, K., Russell, S.: Rao-blackwellised particle filtering for dynamic bayesian networks. In: 16th Conf. on Uncertainty in Artificial Intelligence (2000)

4. Vaswani, N.: Particle filters for infinite (or large) dimensional state spaces-part 2. In: IEEE $\operatorname{ICASSP}(2006)$

5. Rose, C., Saboune, J., Charpillet, F.: Reducing particle filtering complexity for 3D motion capture using dynamic bayesian networks. In: Proc. of AAAI 2008 (2008)

6. Besada-Portas, E., Pliz, S.M., de la Cruz, J.M., Lane, T.: Parallel subspace sampling for particle filtering in dynamic bayesian networks. In: Proc. of ECML 2009 (2009)

7. Koller, D., Lerner, U.: Sampling in factored dynamic systems. In: Sequential Monte Carlo in Practice, pp. 445-464 (2001)

8. Maccormick, J., Blake, A.: A probabilistic exclusion principle for tracking multiple objects. Intenational Journal of Computer Vision 39(1), 57-71 (2000)

9. Klaas, M., de Freitas, N., Doucet, A.: Toward practical $n^{2}$ Monte Carlo: the Marginal Particle Filter. In: Proceedings of UAI 2005 (2005)

10. Doucet, A., Godsill, S., Andrieu, C.: On sequential monte carlo sampling methods for bayesian filtering. Statistics and Computing 10, 197-208 (2000)

11. Fox, D.: Kld-sampling: Adaptive particle filters. In: NIPS 2001 (2001)

12. Soto, A.: Self adaptive particle filter. In: International Joint Conference on Artificial Intelligence (2005)

13. Bolie, M., Hong, S., Djurie, P.M.: Performance and complexity analysis of adaptive particle filtering for tracking applications. In: 36th Conf. on Signals, Sys. and Comput. (2002)

14. Duan, Z., Cai, Z., Yu, J.: Robust position tracking for mobile robots with adaptive evolutionary particle filter. In: Proc. of ICNC 2007 (2007)

15. Nistico, W., Hebbel, M.: Temporal smoothing particle filter for vision based autonomous mobilde robot localization. In: Proc. of ICINCO 2008 (2008)

16. Roefer, T., Jungel, M.: Vision-based fast and reactive monte-carlo localization. In: Proc of ICRA 2003 (2003)

17. Thrun, S., Fox, D., Burgard, W., Dellaert, F.: Robust monte carlo localization for mobile robots. Artificial Intelligence 128 (2001)

18. Burge, J., Lane, T., Link, H., Qiu, S., Clark, V.P.P.: Discrete dynamic bayesian network analysis of fMRI data. Human Brain Mapping (November 2007)

19. Friston, K.J., Harrison, L., Penny, W.: Dynamic Causal Modelling. NeuroImage 19(4) (2003)

20. Lancaster, J.L., Woldorff, M.G., Parsons, L.M., Liotti, M., Freitas, C.S., Rainey, L., Kochunov, P.V., Nickerson, D., Mikiten, S.A., Fox, P.T.: Automated Talairach atlas labels for functional brain mapping. Human Brain Mapping 10(3), 120-131 (2000)

21. Hofmann, R., Tresp, V.: Discovering structure in continuous variables using bayesian networks. In: Advances in Neural Information Processing Systems, vol. 8 (1996) 